\title{
Effects of tachyplesin I on human U251 glioma stem cells
}

\author{
HONG DING $^{1-3 *}$, GANG JIN $^{2 *}$, LIJUN ZHANG $^{2}$, JIANGUO DAI $^{2}$, JIANZHANG DANG $^{2}$ and YALI HAN ${ }^{1}$ \\ ${ }^{1}$ Faculty of Chemical Engineering and Light Industry, Guangdong University of Technology, Guangzhou, Guangdong 510006; \\ ${ }^{2}$ School of Applied Chemistry and Biotechnology, Shenzhen Polytechnic, Shenzhen Public Technology Service Platform for \\ Scale Cell Culture Techniques and Cell Resource, Shenzhen, Guangdong 518055; ${ }^{3}$ Department of Education, \\ Jieyang Vocational and Technical College, Jieyang, Guangdong 522000, P.R. China
}

Received January 24, 2014; Accepted October 2, 2014

DOI: $10.3892 / \mathrm{mmr} .2014 .3021$

\begin{abstract}
Glioblastoma, is one of the most malignant types of intracranial tumor with complex progressive cellular and underlying molecular events. The use of glioma stem cells (GSCs) offers a promising strategy for tumor therapy in the future. Tachyplesin I has been demonstrated to have potential anticancer activity and was first observed in leukocytes. In the present study, the GSC subset was isolated from U251 glioma cells and tachyplesin I was assessed for antitumor activity. As a result, the U251 cells exhibited certain GSC phenotypes, including the expression of stem cell biomarkers CD133 and nestin, when transferred into stem cell culture conditions. The GSCs were grown in an adherent manner in a medium containing serum, while the U251 glioma cells were suspended and cultured in serum-free medium. Tachyplesin I damaged the structure of GSC and inhibited the culture of GSC spheres in a time and dose-dependent manner. When tachyplesin I was administered at a concentration of $10-40 \mu \mathrm{g} / \mathrm{ml}$, GSC differentiation was induced. GSCs treated with a low dose of tachyplesin I disrupted the plasma membrane and led to a loss of cytoplasmic organelles. These findings indicated that tachyplesin I had an effect on inhibiting tumor stem cells and demonstrated that tachyplesin I inhibited GSCs by disrupting the plasma membranes and inducing GSC differentiation.
\end{abstract}

\section{Introduction}

Tachyplesin I is a disulfide-stabilized $\beta$-hairpin antimicrobial peptide with 17 residues that can be isolated from hemocytes of the horseshoe crab (Tachypleus tridentatus). This peptide

Correspondence to: Dr Gang Jin, School of Applied Chemistry and Biotechnology, Shenzhen Polytechnic, Shenzhen Public Technology Service Platform for Scale Cell Culture Techniques and Cell Resource, 2190 Liuxian Road, Nanshan, Shenzhen, Guangdong 518055, P.R. China

E-mail: gangjin1234@yeah.net

*Contributed equally

Key words: tachyplesin I, glioma stem cells, CD133, nestin, differentiation inhibits the growth of Gram-negative and Gram-positive bacteria at particularly low concentrations (1,2). A synthetic peptide from tachyplesin I has been demonstrated to decrease the growth of tumor cells in vitro and in vivo following linkage to the integrin homing arginine-glycine-aspartic acid (RGD) domain (3). Several studies had demonstrated that tachylesin inhibited the proliferation of tumor cells, including gastric adenocarcinoma, human hepatocarcinoma, prostate cancer and melanoma (3-5). In addition, tachyplesin I affects the differentiation of tumor cells (6). These findings suggest that tachyplesin I may be used to as an anti-tumor agent.

Malignant gliomas are aggressive brain tumors with limited therapeutic options. According to the type of glial cell in cancerous tissues, gliomas can be divided into four types, astrocytoma, oligodendroglioma or ependymoma (7). Glioma are composed of heterogeneous types of tumor cells that differ in their expression of markers and growth capacities (8). At present, glioma stem cells (GSCs) have been identified as the major reason for chemotherapy resistance in high-grade glioma (9). In addition, stem-like cells (CD133+) have been found in malignant brain tumors and included cells expressing specific neural progenitor proteins, including nestin, SOX2 and OCT4 $(10,11)$. These could initiate tumor formation following xenotransplantation (12).

The cancer stem cell (CSC) hypothesis provides new insight into the heterogeneity of types of malignant tumor (8). CSCs account for several important processes in carcinogensis, including tumor invasion, angiogenesis and recurrence, and tumor heterogeneity is determined, in part, by the presence of these CSCs $(13,14)$. Therefore, the strategy in tumor therapy is aimed at destroying the tumorigenic CSCs $(14,15)$. The present study aimed to examine the effects of tachplesin I on GSCs cells obtained from glioma U251 cell lines. It was hypothesized that tachplesin I is able to inhibit the proliferation of GSCs from glioma and is a potential antitumor drug.

\section{Materials and methods}

Tachyplesin I synthesis and cell culture. A peptide of tachyplesin containing the sequence $\mathrm{NH}_{2}-\mathrm{K}-\mathrm{W}-\mathrm{C}-\mathrm{F}-\mathrm{R}-\mathrm{V}-\mathrm{C}-\mathrm{Y}-\mathrm{R}-\mathrm{G}-\mathrm{I}-\mathrm{C}-\mathrm{Y}-\mathrm{I}$ -R-R-C-R-CONH${ }_{2}$ was synthesized by Hanyu Bioengineering Company (Shenzhen, China) with a purity of $>95 \%$. There were two disulfide bonds present in two positions, located between the $3 \mathrm{rd}$ and 16 th cysteine and between the 7 th and 12 th cysteine. 
The $\mathrm{NH}_{2}$-terminal was acetylated and the $\mathrm{COOH}$-terminal was amidated to prevent its degradation. Prior to use, the peptides were dissolved in PBS at a concentration of $5 \mu \mathrm{g} / \mu \mathrm{l}$.

U251 human glioma cells were purchased from the Chinese Academy of Sciences Cell Band (Shanghai, China), cultured in RPMI-1640 (Gibco-BRL Life Technologies, Grand Island, NY, USA) and supplemented with $10 \%$ fetal bovine serum (Gibco-BRL), $1 \%$ penicillin and streptomycin (Hyclone, Logan, UT, USA). The U251 cells were collected in the logarithmic growth phase. The U251 cells were thoroughly dissociated using $0.25 \%$ trypsin to prepare single-cell suspensions and then plated onto $10 \mathrm{~cm}$ dishes for culture in serum-free medium at a density of $30-50$ cells $/ \mathrm{cm}^{2}$. Following incubation for 7 days at in 5\% $\mathrm{CO}_{2}$ and $100 \%$ humidity, clones of different morphological types were observed and adherent cells at the bottom were removed. Subsequently, the single-cell suspensions were cultured in 24-well plates at a clonal density and passaged at 1:2 or 1:3.

Self-renewal assay and induction of differentiation. The suspended cells $\left(1 \times 10^{4}\right.$ cells $\left./ \mathrm{ml}\right)$ were cultured in neurobasal-A medium, which consisted of $50 \mathrm{ng} / \mathrm{ml}$ basic fibroblast growth factor (bFGF; Gibco-BRL), $50 \mathrm{ng} / \mathrm{ml}$ epidermal growth factor (EGF, Gibco-BRL) and $10 \mu 1 / \mathrm{ml}$ B27 (Gibco-BRL) or RPMI-1640 supplemented with $10 \%$ fetal bovine serum (Gibco-BRL), 1\% penicillin and streptomycin, and primary clone spheres were formed. Subsequently, primary clone spheres were digested using stem cell accutase (Gibco-BRL), centrifuged at $1,000 \mathrm{x}$ g for $5 \mathrm{~min}$ and single cells were obtained for further culture to form secondary clone spheres.

Immunofluorescence microscopy and dyes. The second or third generation U251 tumor spheres were centrifuged and suspended in complete media. The cells were then dropped onto a polylysine embedding slice and fixed using $4 \%$ paraformaldehyde (Sigma-Aldrich, St. Louis, MO, USA) for $30 \mathrm{~min}$. Following this, the cells were permeabilized with $0.3 \%$ Triton $\mathrm{X}-100$ in phosphate-buffered saline (PBS) for $15 \mathrm{~min}$ and inhibited with $3 \%$ bovine serum albumin for $30 \mathrm{~min}$ before adding the following primary antibodies: Mouse anti-human Nestin (Abcam, Cambridge, MA, USA) and mouse anti-human CD133 (Abcam). The secondary goat anti-mouse immunoglobulin (Ig)G labeled with fluorescein isothiocyanate or cyanine-3 (Abcam) were added. Images were captured using an Olympus fluorescence microscope (Olympus, Toykyo, Japan). The contrast and brightness of the micrographs were then adjusted using Adobe Photoshop CS software (Adobe Inc., San Jose, CA, USA) for data presentation.

Administering of GSC with tachyplesin I and 3-4,5-dimethylthiazol-2-yl-2,5-diphenyltetraolium bromide (MTT) assay. The second or third generation stem cell spheres were administered with tachyplesin I. Following seeding for $24 \mathrm{~h}$, the experimental groups were treated with reagent containing tachyplesin I in a concentration gradient $(0,10,20,40,80$ and $160 \mu \mathrm{g} / \mathrm{ml}$ ). After $24 \mathrm{~h}$, images were captured using an Olympus fluorescence microscope (Olympus).

Cell viability was determined using an MTT assay. Initially, U251 tumor spheres were digested to single cells using $0.25 \%$ trypsin and seeded in a 96 well-plate. Tachyplesin I was used to treat cells when the concentration reached to $10^{3}-10^{4}$ cells in each well. Following treatment with different concentrations of tachyplesin I for 24 or $48 \mathrm{~h}$, the cultures were washed with PBS. Subsequently, MTT $(0.5 \mathrm{mg} / \mathrm{ml})$ was added to each well and the mixture was incubated at $37^{\circ} \mathrm{C}$ for $4 \mathrm{~h}$. Dimethyl sulfoxide (DMSO) of an equal volume was used instead of the culture medium to dissolve the formazan crysals. The mixture was then agitated at room temperature for $10 \mathrm{~min}$ and the absorbance of each well was determined at $490 \mathrm{~nm}$ using a microplate reader (Bio-Tek Instruments, Inc., Winooski, VT, USA). All experiments were repeated six times and the data are expressed as the mean \pm standard deviation (SD). The inhibitory rate of tyachyplesin was assessed using the following formula: inhibitory rate $=($ OD490 value of controls - OD490 value of tachyplesin I-treated U251 cells) / (OD490 value of controls - OD490 value of blanks) x 100\%.

Transmission electron microscopy. The cells were fixed in $0.1 \mathrm{M}$ phosphate buffer ( $\mathrm{pH}$ 7.2) containing $2.5 \%$ glutaraldehyde (Sigma-Aldrich) for $1 \mathrm{~h}$. This was followed by fixation in $0.1 \mathrm{M}$ phosphate buffer ( $\mathrm{pH}$ 7.2) containing $1 \% \mathrm{OsO}_{4}$ (Sigma-Aldrich) for $1 \mathrm{~h}$. The specimens were dehydrated in graded ethanol (Zhongshan Jinqiao, Beijing, China), embedded in epoxy resin (Electron Microscopy Sciences, Hatfield, PA, USA), cut into ultrathin sections and stained using uranyl acetate and lead citrate (Zhongshan Jinqiao). The stained ultrathin sections were observed by transmission electron microscopy (H-9500; Hitachi, Tokyo, Japan).

Scanning electron microscopy. For standard scanning electron microscopy, the cells were fixed in $0.1 \mathrm{M}$ phosphate buffer $(\mathrm{pH}$ 7.2) containing $2.5 \%$ glutaraldehyde for $1 \mathrm{~h}$ and subsequently fixed in $0.1 \mathrm{M}$ phosphate buffer ( $\mathrm{pH}$ 7.2) containing $1 \% \mathrm{OsO}_{4}$ for $1 \mathrm{~h}$. The cells were then dehydrated in graded ethanol and critical-point air drying was performed, following treatment with isomyl acetate. The samples were sputter coated with $\mathrm{OsO}_{4}$ and observed using a scanning electron microscope (S-4800; Hitachi, Tokyo, Japan) (17).

Statistical analysis. Data are expressed as the mean \pm standard deviation. Differences between the mean values for individual groups were assessed using a Wilcoxon signed-rank test. Statistical analyses were performed using GraphPad Prism 5.0 software (GraphPad Software, Inc., La Jolla, CA, USA). P $<0.05$ was considered to indicate a statistically significant difference.

\section{Results}

Serum deprivation in U251 cells induces tumor stem cell sphere formation. Initially, the U251 cells were inoculated with serum-free medium. After $24 \mathrm{~h}$, the U251 cells were adhesively grown with irregular protrusions. No proliferation of the U251 cells was observed (Fig. 1A). However, following culture in serum-free medium supplemented with B27, bFGF and EGF, the U251 tumor cells became non-adhesive and formed tumor spheres after 3 days (Fig. 1B). Subsequently, individual clonal spheres grew suspended in medium in the following 2-3 days. In addition, the majority of the clonal spheres demonstrated spherical or ovoid shapes and the cells exhibited a high proliferative activity. By contrast, the adhesive cells were programmed 

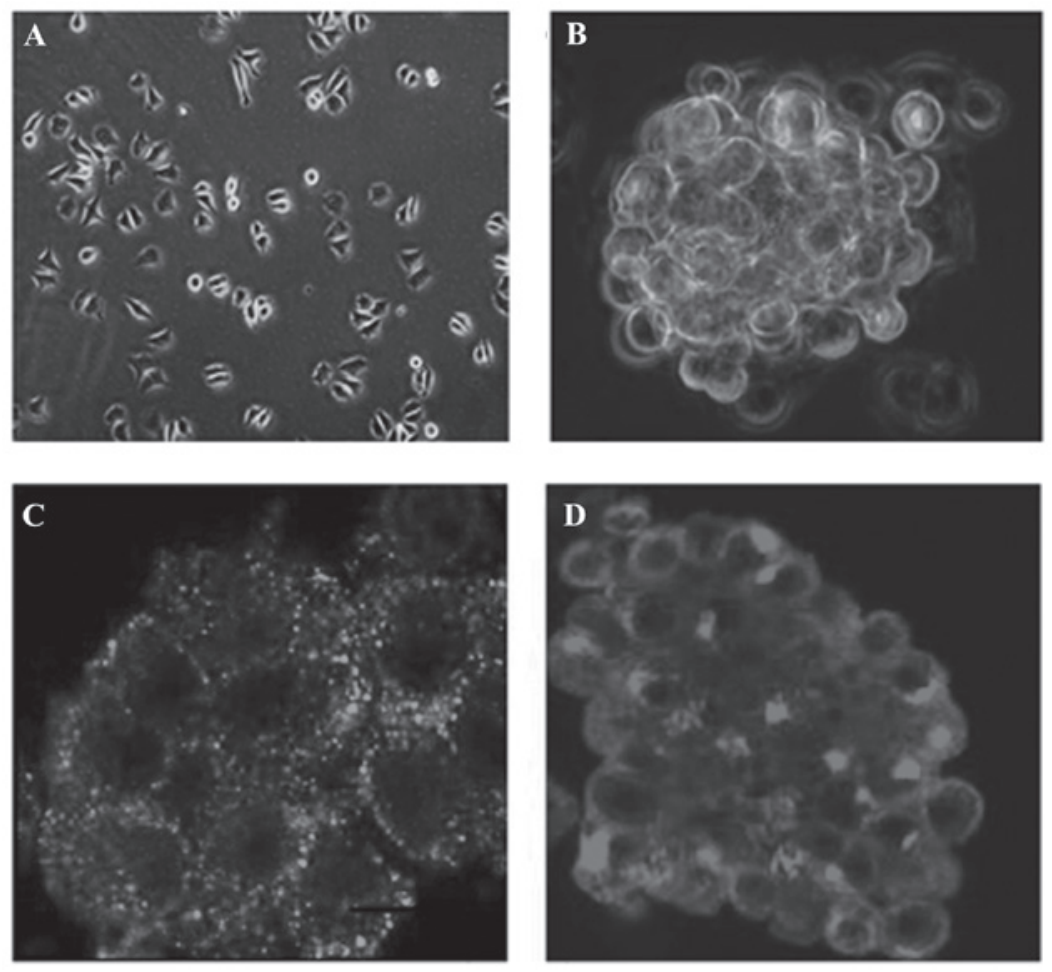

Figure 1. Differing growth patterns of U251 glioma cells cultured in different conditions. (A) U251 cells demonstrated adherent growth in serum-free medium for $24 \mathrm{~h}$. (B) U251 cells demonstrated non-adherent sphere growth in serum-free medium. Immunofluorescenece analysis of the expression of (C) CD133 and (D) nestin. Magnification, x400.
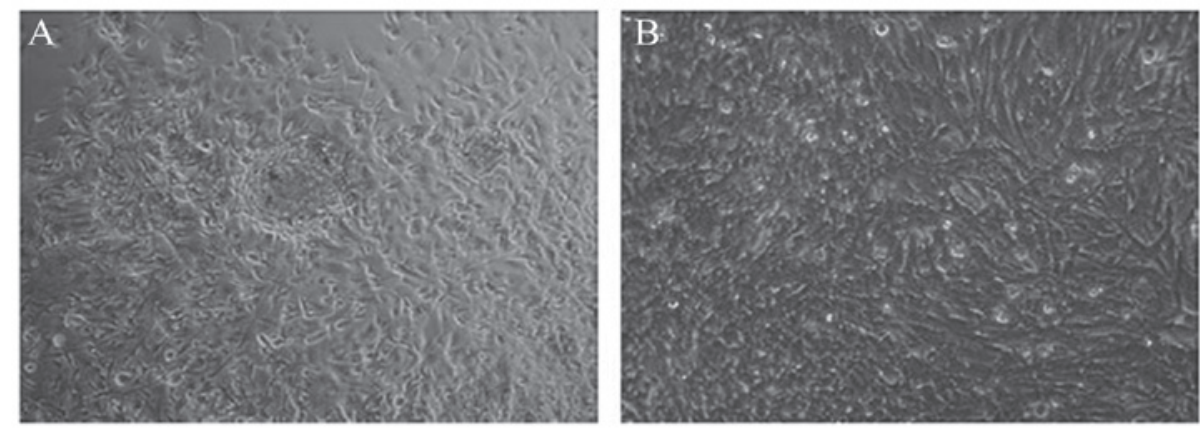

Figure 2. GSCs exhibit neruosphere-type, non-adherent growth when cultured in serum-supplemented media. (A) Morphology of GSC spheres cultured in serum-free media after 24 h. (B) After 72 h, adherent cells are fused, with spindle or stellate morphology. Magnification, x200. GSC, glioma stem cells.

towards cell death. CD133 and nestin, markers of brain CSCs, were detected using indirect immunofluorescence. As shown in Fig. 1C and D, CD133 and nestin were positively expressed in the suspended clone spheres. Based on these results, the suspended spheres derived from U251 cells formed glioma stem cells.

U251 stem cell growth patterns in different media. The U251 stem cells demonstrated different growth patterns in serum and serum-free medium. When isolated GSCs were cultured in medium with serum, after $4 \mathrm{~h}$, the cell spheres grew against the wall of the flask. Following $12 \mathrm{~h}$ culture, the single cells had grown in the periphery of the stem cell spheres. As shown in Fig. 2A, the cells were rounded with some areas of cell flattening $24 \mathrm{~h}$ after culture. Adherent cells formed a monolayer at day 3 (Fig. 2B). No differences were observed in the morphological characteristics of GSCs following culture in serum medium compared with routine methods of $\mathrm{U} 251$ cell culture. However, when GSCs were cultured in serum-free medium, GSCs were observed to grow in a suspended manner with tumor stem-like morphology. In the following consecutive passages, conservation of GSC sphere morphology, characteristics and proliferative capacity was observed. Alterations in the growth pattern of the GSCs coincided with the medium used during culture. In the medium containing serum, the GSCs grew in an adherent manner, while the cells were suspended following culture in serum-free medium. Notably, the GSCs were plastic in response to their environment.

Inhibition of GSC proliferation by tachyplesin I is dose dependent. As GSCs are important in determining tumor progression, the present study hypothesized that the inhibition of GSCs contributed to limiting tumor proliferation. Therefore, 

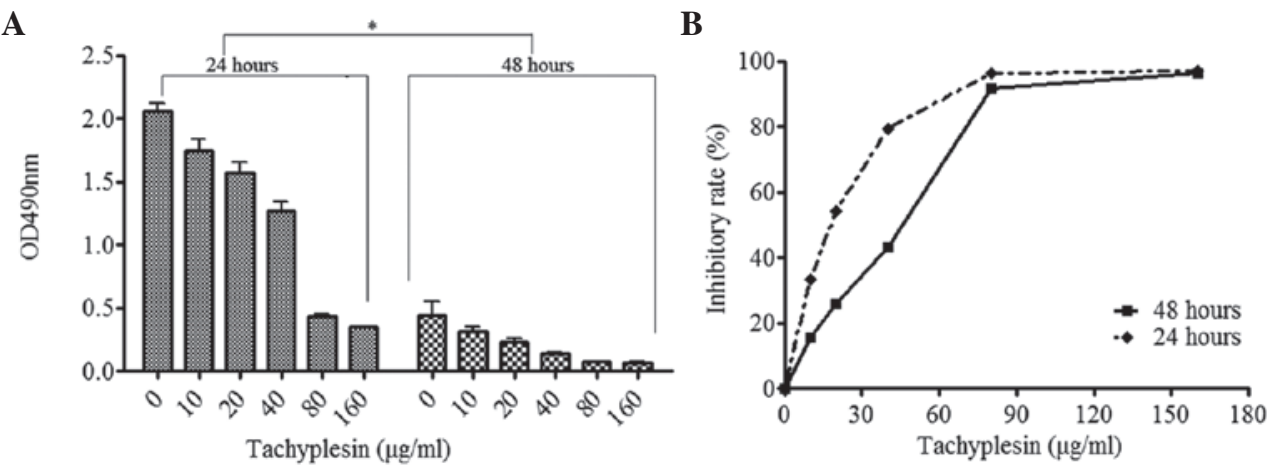

Figure 3. Growth inhibition of U251 GSCs following treatment for 24 or $48 \mathrm{~h}$ with varying concentration of tachyplesin. (A) 3-4,5-dimethylthiazol-2-yl-2,5-diphenyltetraolium bromide assay revealed that tachyplesin inhibited the GSC and U251 cells in a dose- and time-dependent manner ("P<0.05). (B) Inhibitory rate increased in accordance with the elevation in tachyplesin concentration. GSC, glioma stem cells; OD, optical density.

the present study examined the effects of tachyplesin I on the growth of GSCs. An MTT assay was used to evaluate the proliferation of GSC and U251 cells following treatment with various concentrations of tachyplesin I for 24 or $48 \mathrm{~h}$. As shown in Fig. 3A, GSC proliferation was inhibited by tachyplesin I and the OD490 values were lower in the group treated for $48 \mathrm{~h}$ compared with those treated for $24 \mathrm{~h}(\mathrm{P}=0.0313)$. Therefore, in addition to the decrease in OD490 with elevation in tachyplesin I concentration, the OD490 values declined between 24 and $48 \mathrm{~h}$ (Fig. 3A). These results indicated that the effects of tachyplesin I on GSC proliferation occurred in a dose and time-dependent manner. The growth inhibitory rates observed at the concentrations of $10,20,40,80$ and $160 \mu \mathrm{g} / \mathrm{ml}$ were $15.70,25.82,43.24,91.70$ and $96.45 \%$, respectively, $24 \mathrm{~h}$ after treatment. After $48 \mathrm{~h}$, the growth inhibitory rates were 33.47 , $54.12,79.44,96.20$ and $97.16 \%$, respectively. These results demonstrated that tachyplesin I had marked anti-proliferative effects on the GSCs (Fig. 3B). In addition, it was observed that the higher the concentration of tachyplesin I, the stronger the anticancer-effects.

Tachyplesin I damages the structure of GSCs. Following treatment of the GSC spheres with a series of concentrations of tachyplesin I, observation using an inverted microscope after $24 \mathrm{~h}$ demonstrated destruction of the morphological characteristics.(Fig. 4). Furthermore, a notable feature of the brain tumor-initiating cells was the maintenance of tumor cells in an undifferentiated state. For this reason, new therapeutic approaches that force initiating cells to undergo differentiation to cease proliferation is being sought to improve cancer treatment (18). When tachyplesin I was administered at a concentration of 10-40 $\mu \mathrm{g} / \mathrm{ml}$, which induced GSC differentiation, certain cells extended outward from the spheroids in an adhesive manner (Fig 4B-D). As the concentration of tachyplesin I increased to $80 \mu \mathrm{g} / \mathrm{ml}$ (Fig 4E), there were several vesicles within the spheres and the cell contents leaked out. A $160 \mu \mathrm{g} / \mathrm{ml}$ concentration of tachyplesin I induced cell death (Fig. 4F).

Imaging of intracellular deposition of GSCs using transmission and scanning electron microscopy. To investigate the effects of tachyplesin I on GSCs, transmission and scanning electron microscopy were used to examine morphological changes. The primary cells were easily identified by their lack of a nucleolus and small quantities of cytoplasm and organelles (Fig. 5), consistent with stem cells (19). Initially, the GSCs had a clear cell structure (Figs. 5A and 6A) in the absence of tachyplesin I treatment. The nuclei were darkly stained and located in the central area with irregular morphologies. The cytoplasm contained abundant mitochondria, endosomes, Golgi apparati and endoplasmic reticula. The transmission electronmicrograph revealed the inner GSC structures, with the cells containing dendritic processes and folds. However, the cells treated with a low dose of tachyplesin I had a disrupted plasma membrane, which led to the loss of cytoplasmic organelles and a reduction in volume (Figs. 5B-D and 6B-D). Furthermore, a high dose of tachyplesin I promoted cell death (Figs. 5E-F and 6E-F).

\section{Discussion}

Several studies have revealed that the progress of carcinogenesis is driven and possibly accelerated by a subgroup of cancer stem-like cells with higher self-renewal capacity and the ability to differentiate (20). Despite the development of neurosurgery, chemotherapy and radiotherapy in previous decades, the mean survival rate of patients with malignant glioma is limited to 2 years. GSCs may be a potential target to improve the clinical efficacy of chemotherapy (20). In the present study, it was demonstrated that tachyplesin I inhibited tumor growth by inducing GSC differentiation and death.

The separation of stem-like cells from cancer cell lines offers a useful model for reflecting the biological activities present in the human body during carcinogenesis (22). valuable and accurate model of disease is possible by the establishment of tumor cell lines, which retain their stem cell properties to initiate cancer (22). In the present study, a large quantity of GSCs were obtained, which expressed CD133 and nestin, the typical stem cell biomarkers (23). These cells may represent the most malignant subset of tumor-initiating cells (9). The use of MTT assays revealed that tachyplesin I inhibited the proliferation of GSCs in a dose and time-dependent manner. However, due to a lack of sufficient evidence, the detailed mechanism of how tachplesin disrupts the progress of tumor stem-like cells remains to be elucidated. The cationic amino acids of tachyplesin I have been detected in negatively charged 

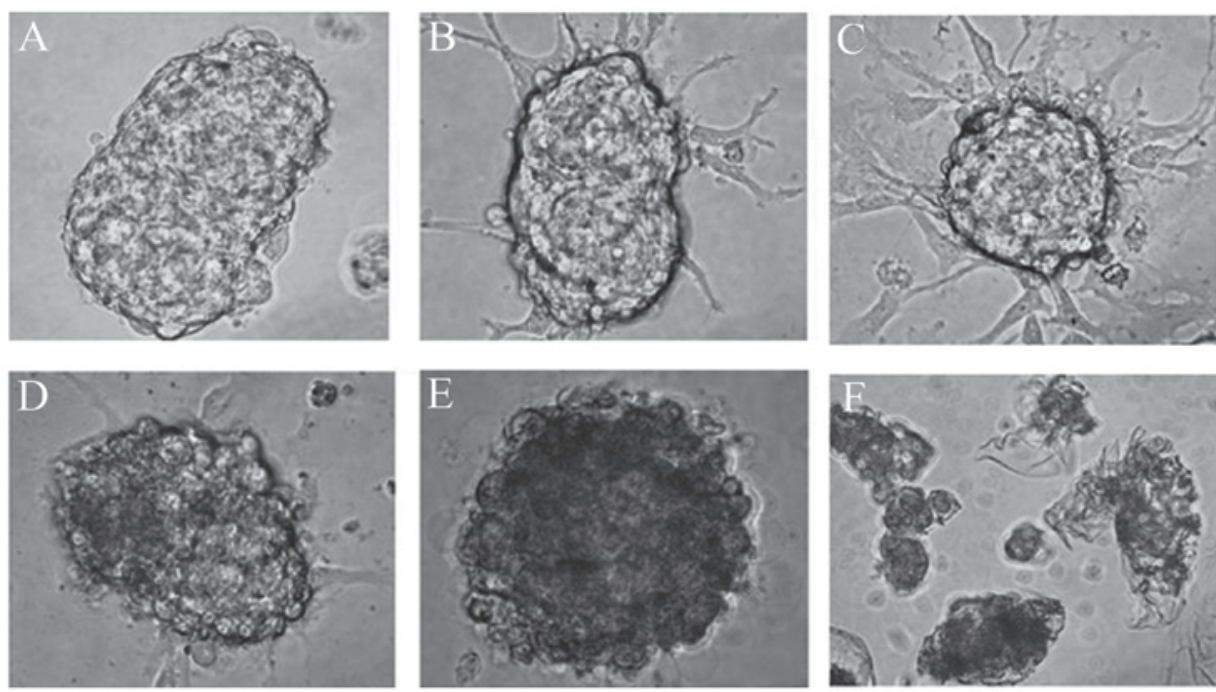

Figure 4. Morphological alterations following treatment with a series of concentrations of tachyplesin I. (A) 0, (B) 10 , (C) 20 , (D) 40 , (E) 80 and (F) $160 \mu \mathrm{g} / \mathrm{ml}$. Magnification, $\mathrm{x} 400$.
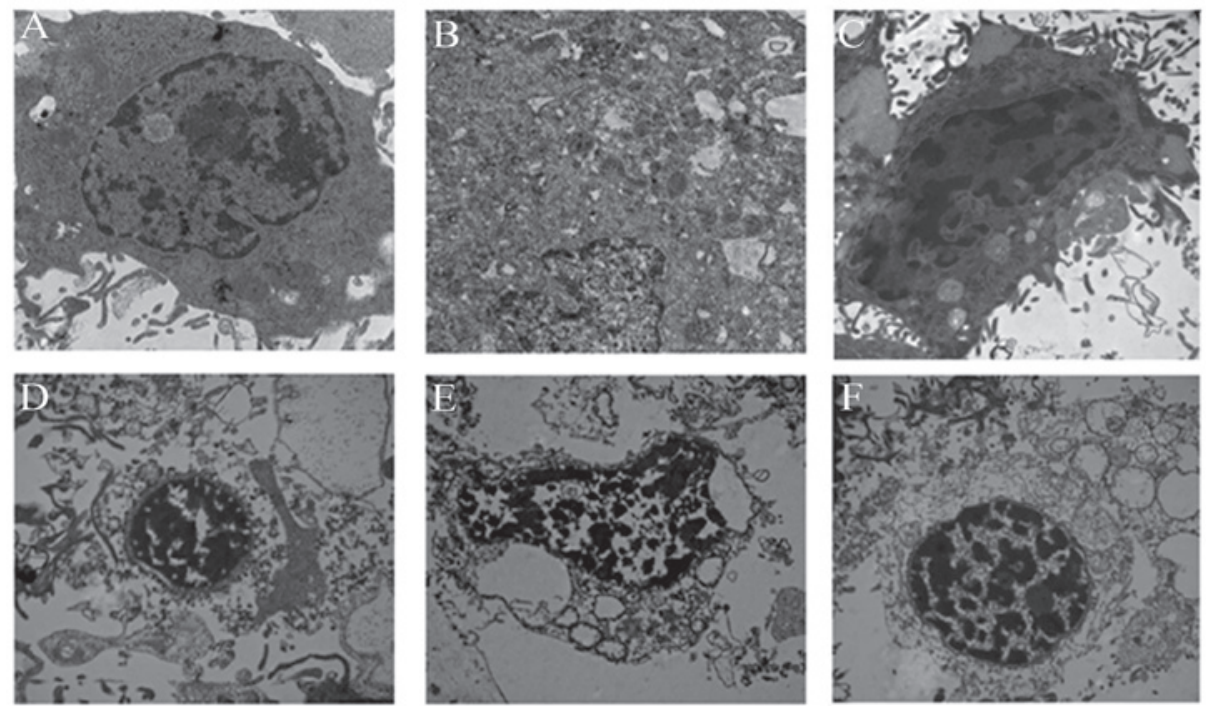

Figure 5. Imaging of glioma stem cell structural alterations following treatment with tachyplesin using transmission electron microscopy. (A) 0 , (B) 10 , (C) 20 , (D) 40, (E) 80 and (F) $160 \mu \mathrm{g} / \mathrm{ml}$ tachyplesin I. Magnification, x20,000.

A

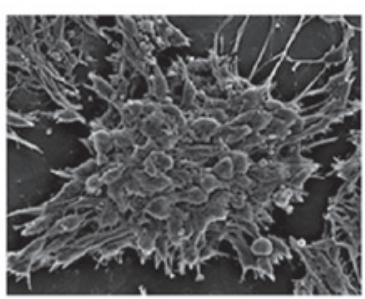

D

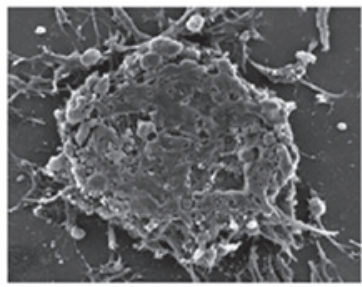

B

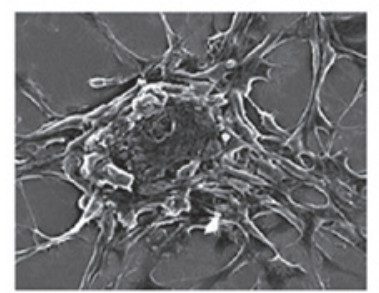

C

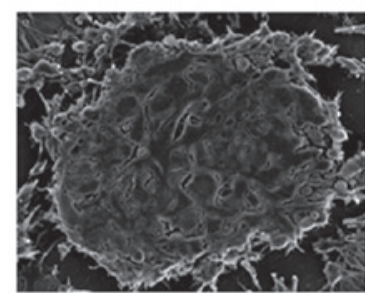

$\mathbf{E}$

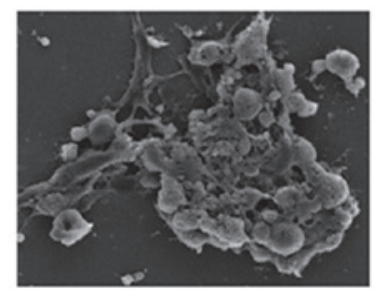

$\mathbf{F}$

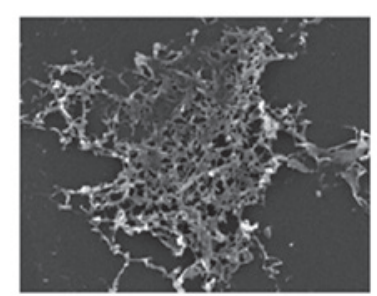

Figure 6. Imaging of glioma stem cells using scanning electron microscopy at different concentrations of tachyplesin I. (A) 0 , (B) 10 , (C) 20 , (D) 40 , (E) 80 and (F) $160 \mu \mathrm{g} / \mathrm{ml}$ tachyplesin I. Magnification, $\mathrm{x} 500$. 
membranes, which interact with anionic head groups of phospholipids (24). In addition, tachyplesin I can destroy the integrity of the membrane by disrupting the lipid bilayer with their amphipathic helices (24). Above all, tachyplesin I is more likely to disrupt prokaryotic and mictochondrial membranes, as compared with the plasma membranes of eukaryotic cells due to the presence of zwitterion phospholipids in eukaryotic cells. The mechanism of the mitochondrial pathway, which induces tumor cell apoptosis is important in inhibiting tumor progression. In previous studies and in the present study, tachyplesin I was found to be important in the disruption of mitochondrial membranes and, therefore, it is proposed that tachyplesin I induces GSC apoptosis. Chen et al demonstrated that RGD-tachyplesin I led to upregulation in apoptosis associated with the mitochondrial and death receptor pathways. RGD-tachyplesin I activated the expression of either caspase 9, 8 and 3 or increased expression of the Fas ligand, and induced cell apoptosis. Furthermore, RGD-tachyplesin I also prevented tumor growth on the chorioallantoic membranes of chicken embryos and in syngeneic mice (3). These results highlight the potential use for tachyplesin I as an anti-tumor agent in clinical treatment in the future.

Tachyplesin I may have other roles in regulating GSC development. In the present study, tachyplesin I was found to induce GSC differentiation. Li et al demonstrated that thachyplesin downregulated the levels of mutant p52, cyclin D1 and CDK4 proteins and c-myc mRNA. It also induced the differentiation of human hepatocarcinoma cells (4) and induced the differentiation of the human hepatocarcinoma cell line, SMMC7721 (25). Furthermore, tachyplesin I not only inhibits the proliferation of tumor cells, but also regulates the cell cycle (4). In a previous study, tachyplesin I arrested the cell cycle at the G0/G1 stage (4) and induced an immune reaction. Chen et al (24) found that classic complement cascade reactions were induced following the linkage of tachyplesin I to hyaluronan in tumor cells or to $\mathrm{Clq}$ in the serum. This resulted in tachyplesin I disrupting the integrity of the tumor cell membrane and inducing cell death (24). Therefore, tachyplesin I has multiple effects in regulating tumor cell growth, including the induction of tumor cell apoptosis, differentiation and cell cycle arrest at the G0/G1 stage.

In conclusion, the present study demonstrated that tachyplesin I inhibited GSCs by disrupting the plasma membranes and inducing GSC differentiation. Further investigation of tachyplesin I, focussing on the detailed mechanisms of its role in inhibiting tumor stem cells is required. In addition, it is necessary to examine the role of tachyplesin I on glioma carcinogensis in vivo.

\section{Acknowledgements}

This study was supported by the Shenzhen Basic Research Key Projects (grant nos. JCYJ20130331151022276, 2111K3070010, JC201005280534A and JC201105201191A), the Guangdong Province Natural Science Foundation (grant nos. 10151805501000007 and S2011020005160), GDPRSFS (2012), GDUHTP (2011 and 2013) and the National Natural Science Funds of China (grant nos. 30800793 and 31272474).

\section{References}

1. Saravanan R, Mohanram H, Joshi M, et al: Structure, activity and interactions of the cysteine deleted analog of tachyplesin-1 with lipopolysaccharide micelle: Mechanistic insights into outer-membrane permeabilization and endotoxin neutralization. Biochim Biophys Acta 1818: 1613-1624, 2012.

2. Doherty T, Waring AJ and Hong M: Dynamic structure of disulfide-removed linear analogs of tachyplesin-I in the lipid bilayer from solid-state NMR. Biochemistry 47: 1105-1116, 2008.

3. Chen Y, Xu X, Hong S, et al: RGD-Tachyplesin inhibits tumor growth. Cancer Res 61: 2434-2438, 2001.

4. Li QF, Ou-Yang GL, Peng XX and Hong SG: Effects of tachyplesin on the regulation of cell cycle in human hepatocarcinoma SMMC-7721 cells. World J Gastroenterol 9: 454-458, 2003.

5. Shi SL, Wang YY, Liang Y and Li QF: Effects of tachyplesin and $\mathrm{n}$-sodium butyrate on proliferation and gene expression of human gastric adenocarcinoma cell line BGC-823. World J Gastroenterol 12: 1694-1698, 2006.

6. Wu XZ and Xie GR: Induced differentiation of hepatocellular carcinoma by natural products. Afr J Tradit Complement Altern Med 5: 325-331, 2008.

7. Eyler CE, Wu Q, Yan K, et al: Glioma stem cell proliferation and tumor growth are promoted by nitric oxide synthase-2. Cell 146: 53-66, 2011.

8. Dalerba P, Cho RW and Clarke MF: Cancer stem cells: models and concepts. Annu Rev Med 58: 267-284, 2007.

9. Gu C, Banasavadi-Siddegowda YK, Joshi K, et al: Tumor-specific activation of the C-JUN/MELK pathway regulates glioma stem cell growth in a p53-dependent manner. Stem Cells 31: 870-881, 2013.

10. Bleau AM, Howard BM, Taylor LA, et al: New strategy for the analysis of phenotypic marker antigens in brain tumor-derived neurospheres in mice and humans. Neurosurg Focus 24: E28, 2008.

11. Mo LJ, Ye HX, Mao Y, et al: B7-H4 expression is high in human U251 glioma stem-like cells and is induced in monocytes cultured in U251 stem-like cell conditioned medium. Chin J Cancer 32: 653-660, 2013.

12. Rahman M, Deleyrolle L, Vedam-Mai V, et al: The cancer stem cell hypothesis: failures and pitfalls. Neurosurgery 68: 531-545, 2011.

13. Singh SK, Clarke ID, Terasaki M, et al: Identification of a cancer stem cell in human brain tumors. Cancer Res 63: 5821-5828, 2003.

14. Vermeulen L, Sprick MR, Kemper K, Stassi G and Medema JP: Cancer stem cells - old concepts, new insights. Cell Death Differ 15: 947-958, 2008.

15. Todaro M,Francipane MG, Medema JP and Stassi G: Colon cancer stem cells: promise of targeted therapy. Gastroenterology 138: 2151-2162, 2010.

16. Vermeulen L, de Sousa e Melo F, Richel DJ and Medema JP: The developing cancer stem-cell model: clinical challenges and opportunities. Lancet Onco 13: e83-e89, 2012.

17. Akita M, Tanaka K, Murai N, et al: Detection of CD133 (prominin-1) in a human hepatoblastoma cell line ( $\mathrm{HuH}-6$ clone 5). Microsc Res Tech 76: 844-852, 2013.

18. Sellheyer K: Stem cell markers can help identify adnexal tumor differentiation when evaluated in the context of morphology: methodology matters. J Cutan Pathol 38: 460-474, 2011.

19. Xue Z, Yan H, Li J, et al: Identification of cancer stem cells in vincristine preconditioned SGC7901 gastric cancer cell line. J Cell Biochem 113: 302-312, 2012.

20. Folkins C, Man S, Xu P, Shaked Y, Hicklin DJ and Kerbel RS: Anticancer therapies combining antiangiogenic and tumor cell cytotoxic effects reduce the tumor stem-like cell fraction in glioma xenograft tumors. Cancer Res 67: 3560-3564, 2007.

21. Yu SC, Xiao HL, Jiang XF, et al: Connexin 43 reverses malignant phenotypes of glioma stem cells by modulating E-cadherin. Stem Cells 30: 108-120, 2012.

22. Pollard SM, Yoshikawa K, Clarke ID, et al: Glioma stem cell lines expanded in adherent culture have tumor-specific phenotypes and are suitable for chemical and genetic screens. Cell Stem Cell 4: 568-580, 2009.

23. Ji B, Chen Q, Liu B, et al: Glioma stem cell-targeted dendritic cells as a tumor vaccine against malignant glioma. Yonsei Med J 54: 92-100, 2013.

24. Chen J, Xu XM, Underhill CB, et al: Tachyplesin activates the classic complement pathway to kill tumor cells. Cancer Res 65: 4614-4622, 2005.

25. Li QF, Ouyang GL, Liu QR and Hong SG: Tachyplesin-induced differentiation of human hepatocarcinoma cell line SMMC-7721. Ai Zheng 21: 480-483, 2002 (In Chinese). 